\title{
Medical Student Ultrasound Education, a WFUMB Position Paper, Part II. A consensus statement by ultrasound societies
}

\author{
Beatrice Hoffmann', Michael Blaivas², Jacques S. Abramowicz ${ }^{3}$, Michael Bachmann Nielsen ${ }^{4}$, \\ Radu Badea ${ }^{5}$, Barbara Braden ${ }^{6}$, Vito Cantisani ${ }^{7}$, Maria C. Chammas ${ }^{8}$, Xin-Wu Cui \\ Yi Dong ${ }^{10}$, Odd Helge Gilja ${ }^{11}$, Roman Hari' ${ }^{12}$, Hein Lamprecht ${ }^{13}$, Harvey Nisenbaum ${ }^{14}$; \\ Christian Pállson Nolsøe ${ }^{15}$, Dieter Nürnberg ${ }^{16}$, Helmut Prosch ${ }^{17}$, Maija Radzina ${ }^{18}$, \\ Florian Recker ${ }^{19}$, Alexander Sachs ${ }^{20}$, Adrian Saftoiu ${ }^{21}$, Andreas Serra ${ }^{22}$, Sudhir Vinayak ${ }^{23}$, \\ Sue Westerway ${ }^{24}$, Yi-Hong Chou ${ }^{25}$, Christoph F. Dietrich ${ }^{26,27}$
}

${ }^{1}$ Harvard Medical School, Department of Emergency Medicine, Beth Israel Deaconess Medical Center, Boston, USA, ${ }^{2}$ University of South Carolina School of Medicine, Department of Emergency Medicine, St Francis Hospital, Columbus Georgia, USA, ${ }^{3}$ Ultrasound Services, Department of Obstetrics and Gynecology, University of Chicago, Chicago, Illinois, USA, ${ }^{4}$ Department of Radiology, Rigshospitalet, University of Copenhagen, Denmark, ${ }^{5}$ Imaging Desk and Ultrasound Department, School of Medicine, University of Medicine and Pharmacy "Iuliu Hatieganu" and Institute of Gastroenterology and Hepatology "Octavian Fodor", Cluj Napoca, Romania, ${ }^{6}$ Translational Gastroenterology Unit, John Radcliffe Hospital, Oxford, UK, ${ }^{7}$ Department of Imaging Diagnostics, Policlinico Umberto I, University Sapienza, Rome, Italy, ${ }^{8}$ Department of Radiology, Hospital das Clinicas, School of Medicine. University of São Paulo, Brazil, ${ }^{9}$ Department of Medical Ultrasound, Tongji Hospital, Tongji Medical College, Huazhong University of Science and Technology, Wuhan, China, ${ }^{10}$ Department of Ultrasound, Zhongshan Hospital, Fudan University, Shanghai, China, ${ }^{11}$ National Centre for Ultrasound in Gastroenterology, Haukeland University Hospital, Bergen and Department of Clinical Medicine, University of Bergen, Norway, ${ }^{12}$ Institute of Primary Health Care (BIHAM), University of Bern, Switzerland, ${ }^{13}$ Division of Emergency Medicine, Stellenbosch University, Cape Town, South Africa, ${ }^{14}$ Department of Medical Imaging, Penn Presbyterian Medical Center, Philadelphia, Pennsylvania, USA, ${ }^{15}$ Centre for Surgical Ultrasound, Dep of Surgery, Zealand University Hospital, Køge Asc Prof, Copenhagen Academy for Medical Education and Simulation (CAMES) University of Copenhagen Denmark, ${ }^{16}$ Brandenburg Medical School Theodor Fontane. Department of Internal Medicine and Gastroenterology, Neuruppin, Germany, ${ }^{17}$ Dept. of Biomedical Imaging and Image-guided Therapy, Medical University of Vienna, Vienna General Hospital, Vienna, Austria, ${ }^{18}$ Diagnostic Radiology Institute, Paula Stradina clinical university hospital, Riga, Latvia; University of Latvia, Radiology Research Laboratory, Riga Stradins University, ${ }^{19}$ Department of Obstetrics and Gynecology, University hospital Bonn, Germany. Sono Education Academy, University Hospital Bonn, Germany, ${ }^{20}$ Department of Biomedical Imaging and Image-guided Therapy. Medical University of Vienna, Vienna, Austria, ${ }^{21}$ Diagnostic and Therapeutic Techniques in Gastroenterology. Research Center of Gastroenterology and Hepatology Craiova, Romania, ${ }^{22}$ Department of Internal Medicine and Nephrology, EFSUMB Learning Center, Klinik Hirslanden, Zürich, Switzerland, ${ }^{23}$ Department of Imaging \& Diagnostic Radiology, WFUMB COE, Aga Khan University Hospital, Nairobi, Kenya, ${ }^{24}$ Ultrasound, Charles Sturt University, NSW, Australia, ${ }^{25}$ Department of Medical Imaging and Radiological Technology, Yuanpei University of Medical Technology, Hsinchu, Taiwan, Department of Radiology, Taipei Veterans General Hospital and School of Medicine, National Yang Ming University, Taipei, Taiwan, ${ }^{26}$ Department Allgemeine Innere Medizin (DAIM), Kliniken Hirslanden Beau Site, Salem und Permanence, Bern, Switzerland, ${ }^{27}$ Ultrasound Department, The First Affiliated Hospital of Zhengzhou University, Zhengzhou, China

Received 18.04.2020 Accepted 03.05.2020

Med Ultrason

2020, Vol. 22, No 2, 220-229

Corresponding author: Prof. Dr. med. Christoph F. Dietrich

Department Allgemeine Innere Medizin (DAIM), Kliniken Hirslanden Beau Site, Salem und Permanence, Bern, Switzerland.

Sino-German Research Center of Ultrasound in Medicine, The First Affiliated Hospital of Zhengzhou University,

Zhengzhou, China

E-mail: c.f.dietrich@googlemail.com; Phone: +41764408150; ORCID ID: orcid.org/0000-0001-6015-6347 


\section{Abstract}

Ultrasound is becoming a fundamental first-line diagnostic tool for most medical specialties and an innovative tool to teach anatomy, physiology and pathophysiology to undergraduate and graduate students. However, availability of structured training programs during medical school is lagging behind and many physicians still acquire all their ultrasound skills during postgraduate training.

There is wide variation in medical student ultrasound education worldwide. Sharing successful educational strategies from early adopter medical schools and learning from leading education programs should advance the integration of ultrasound into the university medical school curricula.

In this overview, we present current approaches and suggestions by ultrasound societies concerning medical student education throughout the world. Based on these examples, we formulate a consensus statement with suggestions on how to integrate ultrasound teaching into the preclinical and clinical medical curricula.

Keywords: ultrasound; skills; education; WFUMB; position paper

\section{Introduction}

The inclusion of ultrasound education into medical school curricula is not a new concept. Some universities have been offering ultrasound education to their medical students for over 20 years, either as part of anatomy or physical diagnosis courses, or during clinical rotation blocks [1,2]. Over the last 2 decades, ultrasound has seen increased clinical use by physicians in many specialties. Mastery of this imaging modality requires the development of spatial aptitude, sono-anatomy and sono-pathophysiology knowledge, as well as an understanding of the limitations of ultrasound. As such, the acquisition of sonographic skills is considered a longitudinal, if not a life-long, learning process. Hence, diagnostic medical sonography should be introduced at the beginning of graduate and postgraduate training [3,4]. As medical education varies significantly among different universities across the world, it would be difficult for every medical school to adapt the exact same approach to teaching ultrasound.

As a general approach, teaching ultrasound can be employed during the pre-clinical and/or clinical years, be web-based, classroom or hospital-based, and consist of theoretical and practical exercises [5-10]. Courses can focus on anatomy or physiology, or can be tailored to medical subspecialties. Three of the most common limitations to introducing ultrasound into a medical school's curriculum appear to be lack of trained instructors, availability of ultrasound machines, and educational opportunities in an already tightly packed medical school curriculum $[11,12]$.

Despite these barriers, ultrasound teaching has found its way into medical school education and has led to a variety of approaches and curricula, frequently supported by regional and national medical societies. In this position paper, we summarize supporting efforts and the current involvement of several Federations and Societies for Ultrasound in Medicine and Biology and some of their collaborators.

\section{The World Federation of Ultrasound in Medicine and Biology (WFUMB) Perspective}

WFUMB's overall purpose is to bring the use of medical ultrasound to every corner of the world. According to the WHO, two thirds of the world's population has no access to medical imaging [10]. WFUMB has a rapidly growing global network of Centers of Education (COE) and presently runs 16 centers in five continental regions. The COEs have two main goals: to focus on the ultrasound education in underserved areas, and to facilitate the dissemination of ultrasound as an integrated part of clinical specialties in the point-of care setting (Point of Care Ultrasound, POCUS). Currently, the main trainee groups of the COEs are medical doctors and other professional healthcare providers. In the future, the work of the COEs will, most likely, increasingly focus on practical student education and integrate collaborative initiatives with local medical schools.

WFUMB is also focusing on improving students' ultrasound education through strengthening collaborations with universities and national student organizations. The goal is to build a curriculum that can be adapted to fit regulations and educational needs in medical schools around the world. One such example is the collaboration between WFUMB and the Society of Ultrasound in Medical Education (SUSME).

WFUMB created a Task Force for Student Education and E-Learning that focuses on the development and distribution of e-learning materials and web-based simulation, as a part of practical training (https://wfumb. info/portfolio-category/e-learning-task-force/). In addition, WFUMB is increasing its student ultrasound education programs at WFUMB congresses. For instance, the WFUMB Congress 2021 in Timisoara will offer a platform for an international student meeting with the aim to train students, students' tutors, discuss different curricula and discuss pathways to integrate e-learning, 
ultrasound phantoms and simulators into a training curriculum.

Furthermore, the WFUMB Education Committee is working on a WFUMB COE Course Book (e-learning material), which would create and collate special teaching material for students' education.

\section{International Federations of Ultrasound in Medicine and Biology}

\section{The Asian Federation of Societies for Ultrasound in Medicine and Biology (AFSUMB) perspective}

The Asian Federation of Societies for Ultrasound in Medicine and Biology (AFSUMB) does not have its own diploma in diagnostic ultrasound, but has been contributing to educational efforts of affiliated national societies by providing qualified speakers/researchers and teams of faculty to join annual meetings and various symposia/workshops. Early examples are the $2^{\text {nd }}$ Ultrasound Workshop of the AFSUMB in Karachi, Pakistan (January $17-19,2004)$ and the $3^{\text {rd }}$ Ultrasound Workshop of the AFSUMB in Shanghai, China (April 9-11, 2005), events at which medical students and trainees were invited to attend with complimentary registration. More current examples include the 2015 AFSUMB's Asian Symposium of Advanced US technology held in Taipei, Taiwan, October 17-18, 2015, and the 2016 Ultrasound Workshop of the AFSUMB in Bangkok, Thailand, November 7-8, 2016, and the 2017 Ultrasound Workshop of the AFSUMB in Beijing, China (August 19-20, 2017), the events at which medical students and trainees were invited to attend with complimentary registration.

AFSUMB also provides scholarships for trainees, either through its organizing committee of its scientific assembly (AFSUMB Congress), or with the support from the Japanese Society of Ultrasound in Medicine (JSUM), Korean Society of Ultrasound in Medicine (KSUM), and Chinese Taipei Society of Ultrasound in Medicine (CTSUM). The CTSUM also provides scholarships for medical students (http://www.sumroc.org.tw/).

\section{The American Institute of Ultrasound in Medicine (AIUM) perspective}

The American Institute of Ultrasound in Medicine (AIUM) is a multidisciplinary professional association and has been advocating for the need for ultrasound in medical school education. It has been pursuing a number of initiatives, ranging from focused conferences and forums to partnering with the Society of Ultrasound in Medical Education (SUSME) on educational events and collaborative projects for medical students [13-16]. AIUM has taken an increasing interest in graduate ultrasound education and has begun holding sessions focused on medical students at its annual conventions, which resulted in an attendance increase by medical students. Additionally, AIUM sponsored conventions to educate United States medical school deans on the importance of ultrasound education in medical school curricula, and conducted workshops on implementation strategies and approaches. These sessions have been well attended by medical school educational leaders and led to the initiation of multiple curricula efforts around the United States.

The AIUM website (AIUM.org) offers free e-learning resources including video tutorials, lectures, training guidelines, a CME tracker, and a toolkit for initiating ultrasound curricula in a medical school setting. These are to assist AIUM members as well as a variety of health professionals in initiating ultrasound curricula in a medical school setting. AIUM also provides connections with mentors in ultrasound education with the goal to help educators dealing with administrative and bureaucratic aspects of introducing ultrasound education into a North American medical school.

\section{The Australasian Society of Ultrasound in Medicine (ASUM) perspective}

ASUM is also a multidisciplinary professional organization with the mission is to promote best ultrasound practice standards.

Of the 22 medical schools in Australia and New Zealand, most have a dedicated radiology / medical imaging component where the theory of ultrasound is taught. The teaching of practical aspects of ultrasound however, is variable among schools. The scope of learning varies from using ultrasound during anatomy classes with cadavers, to another with four half-day practical sessions per year that utilizes local ultrasound practitioners to assist with hands-on instructions for scanning classmates $[17,18]$. Medical students are exposed to ultrasound imaging during their hospital rotations through the various specialties such as obstetrics, cardiology and radiology, although the opportunities for practical experience in these situations is limited.

Most Australasian university health jurisdictions are concerned that medical curriculums are at saturation stage and require a needs assessment and business case before additions to their curriculum are considered. However, ultrasound has been introduced into some universities with success $[17,18]$.

ASUM is a proponent that PoCUS has been shown to assist in improving patient outcomes and as such, it would be of benefit to include ultrasound education for all medical students beginning in the first year of their studies. As with all federations of WFUMB, ASUM will assist, if approached, in formulating ultrasound content. 


\section{European Federation of Societies for Ultrasound} in Medicine and Biology (EFSUMB) perspective

EFSUMB recommends that ultrasound should be used systematically as an easily accessible and instructive educational tool in the curriculum of medical schools [19]. Medical students should acquire theoretical knowledge of the modality, and hands-on training should be implemented adhering to evidence-based principles $[9,10,17]$. The published EFSUMB position paper on student education [19] summarized these current activities, including published teaching materials, standardized examinations, and the EFSUMB e-learning platform.

EFSUMB has launched the Educational Portal on the society website. A textbook is available, the multidisciplinary EFSUMB Course Book (ECB) [20,21]. Each reader is invited and encouraged to improve the ECB, and to optimize the available teaching materials by contacting EFSUMB. These efforts are made to complement and advance the educational purposes of EFSUMB. Furthermore, EFSUMB published the ECB Student Edition (ECBSE), mainly focusing on anatomy, examination techniques, safety issues with accompanying examination technique videos. Specific lessons on US anatomy have been prepared using hands-on live presentations offering self-assessment by the participants [9]. Plans for a future edition to the EFSUMB course book (ECB) include up-to-date ultrasound knowledge, but also providing learning objectives and pre- and posttests. All this material is free to download from the EFSUMB website. The EFSUMB cases of the month and the EFSUMB case atlas serve as an online image database. Additionally, the EFSUMB Task Force on Ultrasound Medical Student Education has recently published a review of the present knowledge and experiences in a short [9] and long version [19]. The aim of the Educational and Professional Standard Committee (EPSC) of EFSUMB is to initiate and monitor international cooperative studies on medical student training in medical ultrasound (mainly in anatomy and physiology) and to provide e-learning resources that can be used for US student education. The key portion was proposed as "anatomy and physiology training of students using ultrasound", with the general objectives of improving anatomy knowledge and the quality of training received by medical school students. The proposal includes specific objectives which are to be reached at the end of each year or cycle of study, and are relative to each medical discipline that benefits from diagnostic ultrasound [10].

The EUROSON Annual Congress contains specific sessions designed for medical students including practical education and discussion with experts. The program of the most recent two EUROSON Congresses integrated training courses in point-of-care ultrasound for students, taught by experienced student tutors reflecting the "nearpeer teaching" concept. Interestingly, several European countries conduct annual meetings of these student peer teachers where teaching curricula and need for student teaching are evaluated.

\section{The Federation of Latin American Societies of Ultrasound (FLAUS) perspective}

The Federation of Latin American Societies of Ultrasound (FLAUS) consists of ultrasound societies all over Latin America (13 countries are members of FLAUS), with the goal of knowledge sharing among its members. Similar to many other countries, in Latin America (LA) ultrasound is performed exclusively by physicians. However, among the members of FLAUS, there is a heterogeneous approach concerning ultrasound education in medical school.

Currently, Latin America has undergraduate and postgraduate ultrasound training available. Both show considerable variability among FLAUS members. Among national universities, there is considerable diversity in the approach to undergraduate ultrasound education. Many universities incorporate ultrasound to a limited degree into the medical student curriculum, frequently in the beginning of medical school. Pathways can take the form of an elective during the second or third year of training and represents a basic introduction to ultrasound. However, some universities have a more advanced and longitudinal approach.

For instance, in 2000, preliminary courses were conducted in Brazil, initially offering extra-curricular courses at the University of São Paulo (USP), at Federal University of São Paulo State (UNIFESP) and Hospital Municipal Souza Aguiar. Some universities in Argentina and Panama have also introduced extra-curricular sonography courses for undergraduate students [22].

The Federal University of Rio de Janeiro School of Medicine started to combine traditional semiology plus point-of-care ultrasound into its curriculum in 2014. In the same year, the Federal University of Juiz de Fora began a program of elective, sequential courses, addressing a variety of sonographic topics. Campinas State University began to offer Emergency Department based courses that included an online Moodle platform and face-toface, competency-based practices. Starting in 2017, the Estácio de Sá University developed the program of integration of ultrasound to the whole curriculum, starting with the disciplines of Propedeutics and Emergency Medicine.

However, the current state of ultrasound teaching in medical school in Latin America as a region is in its beginnings, and there is no published data or consensus on 
the topic for this region. FLAUS is currently working to develop pathways and a certificate for physicians who are performing ultrasound, but were not able to attend a formal radiology residency or regulated ultrasound course.

\section{The Mediterranean and African Society of Ultrasound (MASU) perspective}

MASU is a Society that has been formed to promote the development of ultrasound in the Mediterranean and African regions. The challenges in Africa are immense; both in terms of diversity and extent [23]. Other than a handful of relatively developed radiology services, most regions are struggling with the ability to train physicians. Introduction of simple ultrasound skills to any medical school curriculum would go a long way in ensuring that doctors can perform ultrasound examinations learnt during their undergraduate years, thereby reducing the burden on the scarcely available radiologists [24]. Introducing ultrasound at an undergraduate level will have multiple advantages, ranging from a practical teaching tool, especially for anatomy, to attaining skills to perform ultrasound independently after graduation. However, the main obstacles that prevent ultrasound training in Africa are the cost to purchase machines, technical skill required to maintain these machines, unavailability of skilled instructors, and the logistical support to provide formal training programs [25].

\section{Other Stakeholders}

\section{Society of Ultrasound in Medical Education (SUSME)}

Started more than 9 years ago, SUSME had its first World Congress on Ultrasound in Medical Education in April of 2011. Since that time, SUSME has had multiple other congresses and continues to collaborate with an increasing number of institutions and societies. The mission of SUSME is to promote the use of ultrasound in medical education through development of educational experiences, research on implementation and outcomes, and distribution of results. SUSME is a unique academic society as its focus is exclusively on ultrasound in medical education without having a trade organization-like purpose. Its goal is to be the academic umbrella for ultrasound education and educators throughout the multi-year medical school curriculum. It partners with societies like the American Association of Medical Colleges to achieve ultrasound integration into medical schools, as well as multiple specialty trade organizations. The purpose of the SUSME web site is to:

- provide a forum for the exchange of information about the use of ultrasound in medical education
- facilitate communication and collaboration between educators who are using ultrasound in medical education

- develop a database of information about the use of ultrasound in medical school curricula, and

- provide links to resources, events, and associations related to ultrasound in medicine.

Currently, SUSME is running an international evidence-based ultrasound in medical education consensus conference and delivery of the first consensus statement and publication is expected in the next year. The consensus conference will result in an expected 3 sets of statements over an 18-month period, laying out the best evidence and recommendations for teaching ultrasound in a medical education setting. The consensus panel is made up of over 60 experts acting as voting members and has another layer of consultant representatives numbering nearly 150 international experts. Consensus conference members represent ultrasound education experts from around the world and include leading researchers and educators from nearly all clinical, as well as basic science disciplines. A modified Delphi method is being followed in cooperation with McMaster University in Canada, and is being organized by two expert epidemiologists who have run multiple consensus conferences in the past.

\section{Future Perspective}

Medical education methodology, especially in anatomy, pathophysiology, procedural performance and physical examination, is undergoing a true paradigm shift based on the application of ultrasound technology. This shift will likely accelerate and fundamentally change on how medicine is taught and practiced [16,26]. For future physicians, better knowledge and skills in ultrasound techniques will be pivotal.

Future improvements will include the preclinical integration of ultrasound hands-on education to facilitate the understanding of the main aspects of anatomy, physiology, pathophysiology and pathology, whilst avoiding overloading the student with excessive information. Most common and simple medical scenarios will be used to illustrate the diagnostic value of ultrasound. An internet-based communication platform (forum) between the teachers and the students should be initiated $[9,10]$.

Ultrasound teaching is largely skill based, therefore it requires hands-on training to achieve competency. Ultrasound teaching should also be evaluated as a research tool to assess new didactic and teaching concepts. Worldwide, there is a growing enthusiasm for education and debate about peer-to-peer teaching which brings the question of insufficient teacher-force or use of simula- 
tors for medical students. Thus, it is timely to propose a consistent and structured implementation of ultrasound curricula into medical schools. The highest ranked, most efficient, and vetted curricula should serve as the basic starting point for future standardization with main attention to practical skills [26-28].

Based on the experience and the evidence obtained from using different ultrasound programs in different settings world-wide, the authors involved in this review propose the following statements to be considered regarding course structure, didactic means, equipment of skills labs and the integration into medical curricula.

\section{Example of course structure}

Different examples of a course structures exist, and can be a combination of both online and hands-on learning during a period of 1-3 months. Potential content includes small-group practical training, online tests, formative assessment and case-based discussions

\section{Preparation and pre-course test}

Preparation is necessary to optimize the personal educational benefit of the course. Detailed knowledge of anatomy or intensive repetition represents the most important preparation. In postgraduate courses, additionally knowledge of common and emergency pathologies is supportive.

Physics of ultrasound, documentation, reporting technique, training of visual skills or dexterity are secondary. Though supportive, they have to be part of every handson ultrasound course.

Pre- and post-tests, in other words continuous assessment of sono-anatomy and ultrasound related clinical knowledge, increases learning benefit and performance in professional practice [29].

Apart from synchronous case-based teaching, asynchronous E-Learning before, in between, and after helps to prepare, reflect and deepen knowledge. Concerning the efficiency of learning, usage of different learning modalities, such as listening to presentations, reading learning material, watching instructional videos, casebased learning and practical training, allows participants to reach up to $75 \%$ efficient teaching. Preparation for course directors is concomitantly important. Participants have to be "caught up" at their level of skills, theoretical and practical, following integration into the participant's team to spread enthusiasm of teachers amongst the participants. Preclinical ultrasound courses aim to better understanding of anatomy and physiology. Consequently, precourse questions help to select groups of participants with comparable level of skills, as well as suitable preparation and knowledge to the assigned teacher.
Medical students should have study materials available which best prepares them for hands-on components of the course, including examination techniques, anatomy, and safety issues. A pre-course test is optional, but might motivate students more.

\section{Practical skills, hands-on}

1) Hands-on training via ultrasound skills labs is an important part of any course (more than $50 \%$ of time should be allocated for this activity).

2) Outside of formal courses, extra peer teaching by student tutors and self-training should be encouraged for skill development.

3) Additional supervised hands-on practice during clinical rotations should be accessible.

4) Opportunities for periods of 2-3 weeks for continuous hands-on practice in clinical or imaging settings should be made available.

For hands-on ultrasound training in undergraduate education, we have the following recommendations:

a. Students should be introduced to hands-on skill training as early as possible.

b. Students should practice scanning with different equipment and with different patient positions.

c. Students should train in diverse student groups (different constitutions, prepared vs. unprepared).

d. Students should have the possibility of continuous assessment of practical teaching.

i. Units of $90 \mathrm{~min}, \max 180 \mathrm{~min}$ in one session.

ii. Weekly repetition as continuous as possible.

iii. Enough breaks.

e. Students should practice structured scanning as per adapted guidelines.

f. Students should have access to common and frequent pathologies pertinent to their skill level.

g. Students should master documentation techniques using the US machine (picture vs. video, annotations, button presets).

h. Students should practice how to report regular anatomy.

i. In advanced training levels, students should practice fast vs. slow examinations.

j. In advanced training levels, students should have the possibility to perform supervised patient scanning in clinical settings.

k. After basic practical teaching, students should have the possibility to access non-supervised training settings such as a USSL.

\section{Ultrasound skills lab (USSL) example}

Skills labs provide special practical training aimed at developing the students' hands-on skills in a comfortable learning atmosphere. These utilize special facilities to improve practical skills in examination (e.g. 
physical examination, blood drawing, auscultation of heart and lung, etc.). The Centre for Medical Skills and Simulation should incorporate an Ultrasound Skills Lab (USSL). USSL areas should have special equipment for US hands-on training and US e-learning. In the USSL, different US machines (3-4 traditional and POCUS machines), as well as simulators and phantoms are ideally available for hands-on experience. The students' optimal ratio could be 2-3 at one training station if resources allow. These modules are taught by faculty trainers (professors), student tutors (peer teaching), and by themselves via self-study. Furthermore, the USSL is equipped with anatomical material, US books, charts/posters, e-learning material and access to resources for image review. Hands-on training in the USSL should follow a well-defined schedule based on the main curriculum. Coordination of the USSL should be performed interdisciplinary by enthusiastic clinicians with fundamental knowledge of practical ultrasound in their medical specialty and advanced teaching skills.

\section{Near-peer teaching in US education}

Lack of teaching capacity is a major barrier to the implementation of ultrasound in undergraduate curricula [28]. Near-peer teaching has been evaluated in multiple intervention studies and has shown to be non-inferior to faculty-led teaching e.g. in musculoskeletal [30] or abdominal [31] ultrasound. Near-peer teaching may even have advantages over faculty-led teaching, e.g. the small distance between students and teachers both socially and intellectually that might foster learning because of cognitive and social congruence [32]. Based on the available evidence and the experience from different ultrasound societies, we generally encourage the involvement of near-peer teachers in undergraduate ultrasound training and highlight the importance of a high-quality and continuous teaching for near-peer tutors.

\section{Post Course Test}

Skills taught in ultrasound courses should be subject to a post course examination. Tests can be administered as an Objective Structured Clinical Examination (OSCE), examples include the Objective Structured Assessment of Ultrasound Skills (OSAUS) scale [33] and the OSCE stations validated by Hofer et al [34]. Furthermore, e-learning pathway exams may help for continuous reevaluation of students' knowledge. Due to flexibility and location independent usage, e-learning assessment should be integrated adapted to curriculum milestones. During the study the collection of all passed tools, trainings, courses and lectures in US education in a US students logbook is useful. Evaluation of different US education tools is necessary and helpful implementation of US curricula. Several simulators contain modules that allow evaluation of the student's progress and proficiency in obtaining correct images, either by direct, real-time feedback, or by comparing the student's image with images from an image Library.

\section{The use of guidelines in teaching ultrasound}

Over the last decade WFUMB and its federation of societies produced a series of guidelines and recommendations regarding different ultrasound applications for improved medical education It is imperative for medical ultrasound education to ensure that the teaching is introduced and conducted appropriately based on the best available evidence. Experiences among institutions engaged in ultrasound education should be shared and guidelines and basic recommendations for ultrasound in medical student curricula should be established based on well-designed outcome studies, expert recommendations, and adult learning principles [26].

\section{Potential equipment and resources}

\section{Ultrasound equipment}

Technological advancements have made equipment accessible and affordable, with hand-held ultrasound devices as small as a smartphone [35-37]. Moreover, handheld ultrasound has the potential to enhance the outcome of the physical examination, validating the practitioner's initial clinical findings [10]. It is suggested that the quality of the ultrasound image determines the result of the examination, but for teaching students, the small, inexpensive portable ultrasound devices seem to be sufficient. For demonstration and training in more advanced clinical concepts such as contrast enhanced ultrasound (CEUS) or elastography, a high-quality unit will give a clearer demonstration. In order to understand the full bandwidth of scanning possibilities, US machines with all kinds of probes are mandatory in undergraduate education. Modern solutions with integrated technique in US probes as well as different solutions of image acquisition (piezo crystal vs. semiconductor based) are available in undergraduate US education and introduce early the technical creativity of ultrasound equipment, clinicians will have to work with.

\section{Resources}

The funding of ultrasound education programs remains a crucial aspect of implementation and sustainability, and will depend on the economic resources of the region in which the program is to be implemented. Under resourced medical schools may be extremely challenged to implement such courses, but may qualify for international grants that could support an ultrasound train- 
ing program or might attract sponsorship from industry [26]. Other schools may find avenues to fund programs via gifts or donations, or an increase in student fees to fund the relevant program. Establishing an enthusiastic team of students, clinicians and professors dedicated to US teaching in an appropriate learning atmosphere, e.g. USSL, may be just as important as financial aspects.

\section{Approaches to Data Storage and Reporting}

Documentation tools in medical education have been suboptimal for some time [38]. Data storage and reporting are of utmost importance for quality control and constructive feedback $[12,16]$. This can be achieved by using static images obtained by a video printer, stored images or preferably recorded cine loops. Documentation and reporting should be prepared according to standard terminology. Reporting can be done onsite or remotely by teleradiology. Teleradiology requires adequate bandwidth as stored images are large even when compressed and the appropriate software and communication infrastructure. Third party systems are also available and are often specifically designed for education allowing easy video and image review, feedback and annotation through a remote web interface. There are various types of file compression, but all are either lossy or lossless. Using lossy compression, the original images are not recovered exactly, whereas lossless compression techniques recover exact images of the original images. Various compression algorithm like Lossless JPEG, JPEG-LS, JPEG 2000, PNG and CALIC are in use but JPEG-LS has been found to be the best algorithm based on compression speed and compression ratio for medical image sequences [39].

Future developments in ultrasound image transfer and case discussion could include ultrasound real-time direct live communication and real time ultrasound image transfer. However, privacy and online security of protected health information data will be an important factor in the development of such online and real-time teaching and training options.

Video recording and archiving of entire ultrasound examinations requires huge data storage capacity, but has become possible. This is a valuable tool in teaching, as it allows offline reading and detailed feedback on quality standards and image improvement away from a potential hectic clinical environment [40].

Students should learn and practice precise and accurate reporting using correct terminology. Reports and worksheets should guide the student through a structured approach and use of ultrasound reporting software systems may provide this learning experience. These clini- cal picture archiving and communication databases are searchable and may provide additional educational options for topic-related, case-based group teaching. However, these databases require a high initial expense and constant expensive maintenance, and are not universally available.

\section{Perspectives for postgraduate ultrasound training}

Further training can be determined by the (sub-) specialty the student/physician will be working in. Practicing ultrasound is a life-long learning process and constant refinement of techniques, adaptation to novel technologies/applications as well as advancing clinical knowledge. Regional, national and international networks and tutorships should support further individual progress and learning under the umbrella of the ultrasound societies. Establishing quality indicators in reporting, image documentation and procedure outcomes and defining minimum activity numbers might help raise the general standard of ultrasound performance and to make it progressively comparable.

\section{Summary}

We have discussed current knowledge of ultrasound teaching in medical education including various methodologies and various educational systems. The authors, representing multiple societies and specialties throughout the world, summarize that medical student education and teaching efforts vary greatly among federations. It would be an unrealistic expectation that every medical school adapts the exact same approach to teaching ultrasound.

We encourage the following concepts.

1) To introduce medical student ultrasound education as early as possible in the curriculum, e.g. during preclinical anatomy teaching.

2) To foster acquisition of practical ultrasound skills in an early stage of training (at least 40 hours during early years of medical school training).

3) Skills taught in ultrasound courses should be subject to a post-course examination.

4) The basic teaching content should be examination technique, anatomy (normal findings) and safety issues.

5) To teach standardized medical sonography terminology.

6) To teach so-called "very important pathologies (VIP) is motivating but should be optional.

Conflict of interst: none 


\section{References}

1. Teichgraber UK, Meyer JM, Poulsen Nautrup C, von Rautenfeld DB. Ultrasound anatomy: a practical teaching system in human gross anatomy. Med Educ 1996;30:296-298.

2. Hoffmann B. The future is not the sonoscope. J Ultrasound Med 2003;22:997-998; author reply 998-1000.

3. Solomon SD, Saldana F. Point-of-care ultrasound in medical education--stop listening and look. N Engl J Med 2014;370:1083-1085.

4. Dietrich CF, Hoffmann B, Abramowicz J, et al. Medical Student Ultrasound Education: A WFUMB Position Paper, Part I. Ultrasound Med Biol 2019;45:271-281.

5. Barry DS, Marzouk F, Chulak-Oglu K, Bennett D, Tierney P, O'Keeffe GW. Anatomy education for the YouTube generation. Anat Sci Educ 2016;9:90-96.

6. Tim S: Understanding medical education: evidence, theory, and practice. In: Chichester, ed.: Wiley-Blackwell Publishing, 2010.

7. Hempel D, Haunhorst S, Sinnathurai S, et al. Social media to supplement point-of-care ultrasound courses: the "sandwich e-learning" approach. A randomized trial. Crit Ultrasound J 2016;8:3.

8. Swamy M, Searle RF. Anatomy teaching with portable ultrasound to medical students. BMC Med Educ 2012;12:99.

9. Cantisani V, Dietrich CF, Badea R, et al. EFSUMB statement on medical student education in ultrasound [short version]. Ultraschall Med 2016;37:100-102.

10. Hussain S. Welcome to the Journal of Global Radiology. J Glob Radiol 2015;1.

11. Metzger MJ, Flanagin AJ. Using Web 2.0 technologies to enhance evidence-based medical information. J Health Commun 2011;16 Suppl 1:45-58.

12. Hoppmann RA, Rao VV, Poston MB, et al. An integrated ultrasound curriculum (iUSC) for medical students: 4-year experience. Crit Ultrasound J 2011;3:1-12.

13. Baltarowich OH, Di Salvo DN, Scoutt LM, et al. National ultrasound curriculum for medical students. Ultrasound Q 2014;30:13-19.

14. Chiem AT, Soucy Z, Dinh VA, et al. Integration of Ultrasound in Undergraduate Medical Education at the California Medical Schools: A Discussion of Common Challenges and Strategies From the UMeCali Experience. J Ultrasound Med 2016;35:221-233.

15. Dinh VA, Fu JY, Lu S, Chiem A, Fox JC, Blaivas M. Integration of Ultrasound in Medical Education at United States Medical Schools: A National Survey of Directors' Experiences. J Ultrasound Med 2016;35:413-419.

16. Dinh VA, Lakoff D, Hess J, et al. Medical Student Core Clinical Ultrasound Milestones: A Consensus Among Directors in the United States. J Ultrasound Med 2016;35:421434.

17. Stringer MD, Duncan LJ, Samalia L. Using real-time ultrasound to teach living anatomy: an alternative model for large classes. N Z Med J 2012;125:37-45.

18. Ang J, Doyle B, Allen P, Cheek C. Teaching bedside ultrasound to medical students. Clin Teach 2018;15:331-335.
19. Cantisani V, Dietrich CF, Badea R, et al. EFSUMB Statement on Medical Student Education in Ultrasound [long version]. Ultrasound Int Open 2016;2:E2-7.

20. Dietrich CF, Rudd L, Saftiou A, Gilja OH. The EFSUMB website, a great source for ultrasound information and education. Med Ultrason 2017;19:102-110.

21. Dietrich CF, Rudd L. The EFSUMB website, a guide for better understanding. Med Ultrason 2013;15:215-223.

22. Miner B, Purdy A, Curtis L, et al. Feasibility study of firstyear medical students identifying cardiac anatomy using ultrasound in rural Panama. World J Emerg Med 2015;6:191195.

23. Salmon M, Landes M, Hunchak C, et al. Getting It Right the First Time: Defining Regionally Relevant Training Curricula and Provider Core Competencies for Point-ofCare Ultrasound Education on the African Continent. Ann Emerg Med 2017;69:218-226.

24. LaGrone LN, Sadasivam V, Kushner AL, Groen RS. A review of training opportunities for ultrasonography in low and middle income countries. Trop Med Int Health 2012;17:808-819.

25. Denny SP, Minteer WB, Fenning RTH, et al. Ultrasound curriculum taught by first-year medical students: A four-year experience in Tanzania. World J Emerg Med 2018;9:3340.

26. Hoppmann RA, Rao VV, Bell F, et al. The evolution of an integrated ultrasound curriculum (iUSC) for medical students: 9-year experience. Crit Ultrasound J 2015;7:18.

27. Rempell JS, Saldana F, DiSalvo D, et al. Pilot Point-of-Care Ultrasound Curriculum at Harvard Medical School: Early Experience. West J Emerg Med 2016;17:734-740.

28. Mullen A, Kim B, Puglisi J, Mason NL. An economical strategy for early medical education in ultrasound. BMC Med Educ 2018;18:169.

29. Heinzow HS, Friederichs H, Lenz P, et al. Teaching ultrasound in a curricular course according to certified EFSUMB standards during undergraduate medical education: a prospective study. BMC Med Educ 2013;13:84.

30. Knobe M, Munker R, Sellei RM, et al. Peer teaching: a randomised controlled trial using student-teachers to teach musculoskeletal ultrasound. Med Educ 2010;44:148-155.

31. Celebi N, Zwirner K, Lischner U, et al. Student tutors are able to teach basic sonographic anatomy effectively - a prospective randomized controlled trial. Ultraschall Med 2012;33:141-145.

32. Ten Cate O, Durning S. Dimensions and psychology of peer teaching in medical education. Med Teach 2007;29:546552.

33. Tolsgaard MG, Todsen $\mathrm{T}$, Sorensen JL, et. International multispecialty consensus on al how to evaluate ultrasound competence: a Delphi consensus survey. PLoS One 2013;8:e57687.

34. Hofer M, Kamper L, Sadlo M, Sievers K, Heussen N. Evaluation of an OSCE assessment tool for abdominal ultrasound courses. Ultraschall Med 2011;32:184-190.

35. Mirabel M, Celermajer D, Beraud AS, Jouven X, Marijon E, Hagege AA. Pocket-sized focused cardiac ultra- 
sound: strengths and limitations. Arch Cardiovasc Dis 2015;108:197-205.

36. Barreiros AP, Cui XW, Ignee A, De Molo C, Pirri C, Dietrich CF. EchoScopy in scanning abdominal diseases: initial clinical experience. Z Gastroenterol 2014;52:269-275.

37. Gilja OH, Piscaglia F, Dietrich C. EFSUMB - European Course Book: Echoscopy - A new concept in mobile ultrasound. 2014:1-21.
38. Frohlich E, Hofmann J, Debove I, et al. [Pictocam instead of Pictogram - a quality improvement study in abdominal ultrasound imaging]. Z Med Phys 2016.

39. Ferni M, Umamageswari A, Suresh G. A Survey on Lossless Compression for Medical Images. IJCA 2011;31:0975-8887.

40. Dormagen JB, Gaarder M, Drolsum A. Standardized cineloop documentation in abdominal ultrasound facilitates offline image interpretation. Acta Radiol 2015;56:3-9. 